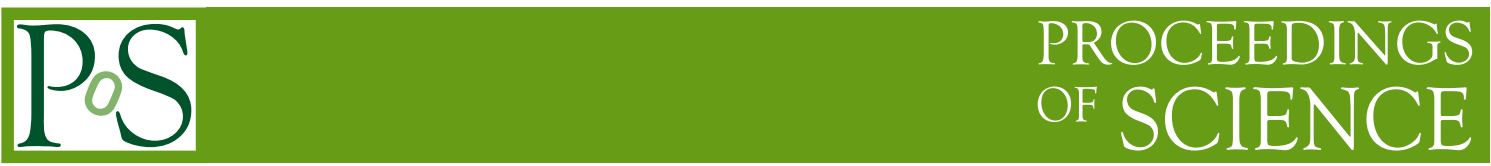

\title{
Charmonium Production and HF decay properties at ATLAS
}

\author{
Andreas Korn, for the ATLAS Collaboration* \\ Department of Physics \& Astronomy, University College London, Gower Street, LONDON, \\ WC1E 6BT \\ E-mail: a.korneucl.ac.uk
}

The ATLAS collaboration has measured the production of heavy flavoured bottom and charm hadrons in proton-proton collisions at the Large Hadron Collider (LHC) at centre of mass energies of $\sqrt{s}=5.02,7,8$ and $13 \mathrm{TeV}$. These measurements serve as an important test of QCD calculations. Production measurements of $J / \psi, \psi(2 S), X(3872)$ charmonium states, as well as $b$-hadron and $J / \psi$ pairs are presented.

XIV International Conference on Heavy Quarks and Leptons (HQL2018)

May 27-June 1, 2018

Yamagata Terrsa, Yamagata,Japan

${ }^{*}$ Speaker. 


\section{Introduction}

Measurements of Heavy Flavour production provides important tests of Quantum Chromodynamics (QCD). The large mass scale of heavy charm and beauty quarks helps to separate perturbative and non-perturbative contributions and hence aides in calculations. Presented here are measurements by the ATLAS detector [1] utilising proton-proton collisions at the Large Hadron Collider (LHC) [2] at centre of mass energies of $\sqrt{s}=5.02,7,8$ and $13 \mathrm{TeV}$.

\section{Bottom production}

The identification of bottom quarks in jets is an important experimental technique at the LHC. Many known and predicted particles decay into $b$-quarks. The identification usually makes use of the relatively long lifetime $\tau$ of the formed $b$-hadrons with an average of $c \tau \sim 450 \mu \mathrm{m}$. The decay vertices of $b$-hadrons and the charged-particle tracks originating from them are displaced from the primary vertex of the collision. These displaced tracks and vertices can be reconstructed and identified. The $b \bar{b}$ production cross section has been measured using such techniques [3].

\subsection{B-hadron pair production}

Current calculations of $b \bar{b}$ production have some disagreements with the data, in particular at small separation angles between the two quarks. As strong $b \bar{b}$ production is a frequent process it also forms an important background to $H \rightarrow b \bar{b}$ decays. In the best reconstructed modes, the Higgs boson will carry a sizeable momentum and hence the resulting $b$-jets in the decay will be relatively close together. Measuring this particular phase space is very difficult with jets due to their limited resolution and relatively large extent. A measurement with partially reconstructed $b$-hadron pairs has a better pointing resolution. The first $b$-hadron is reconstructed in $B \rightarrow J / \psi+X$ decays, while the second utilises $B \rightarrow \mu+Y$ decays. The non-prompt component from $B \rightarrow J / \psi+X$ decays is separated from prompt $J / \psi$ production via a lifetime fit, as shown in Fig. 1. This analysis technique allows to measure the differential $b \bar{b}$ cross section down to an opening angle of zero [4]. The resulting differential cross section is compared with various Monte Carlo generators in Fig. 2. The best description is given by the PYTHIA8 generator with the default setting 4, a transverse momentum based splitting kernel [5].

\section{Charm production}

Due to the shorter lifetime the inclusive reconstruction of charm hadron decays is more difficult. The reconstruction of exclusive decays of charmonium states $\left(J / \psi \rightarrow \mu^{+} \mu^{-}, \psi(2 S) \rightarrow\right.$ $\left.\mu^{+} \mu^{-}, \psi(2 S) \rightarrow J / \psi \pi^{+} \pi^{-}, X(3872) \rightarrow J / \psi \pi^{+} \pi^{-}\right)$provides an alternative.

\section{$3.1 J / \psi \& \psi^{\prime}$ production}

A measurement of $J / \psi \& \psi^{\prime}$ production has to take several sources into account. In particular there is a feed-down of charmonium from $b$-hadron decays. This feed-down can be separated statistically using the pseudo-lifetime of the reconstructed decay vertex. 

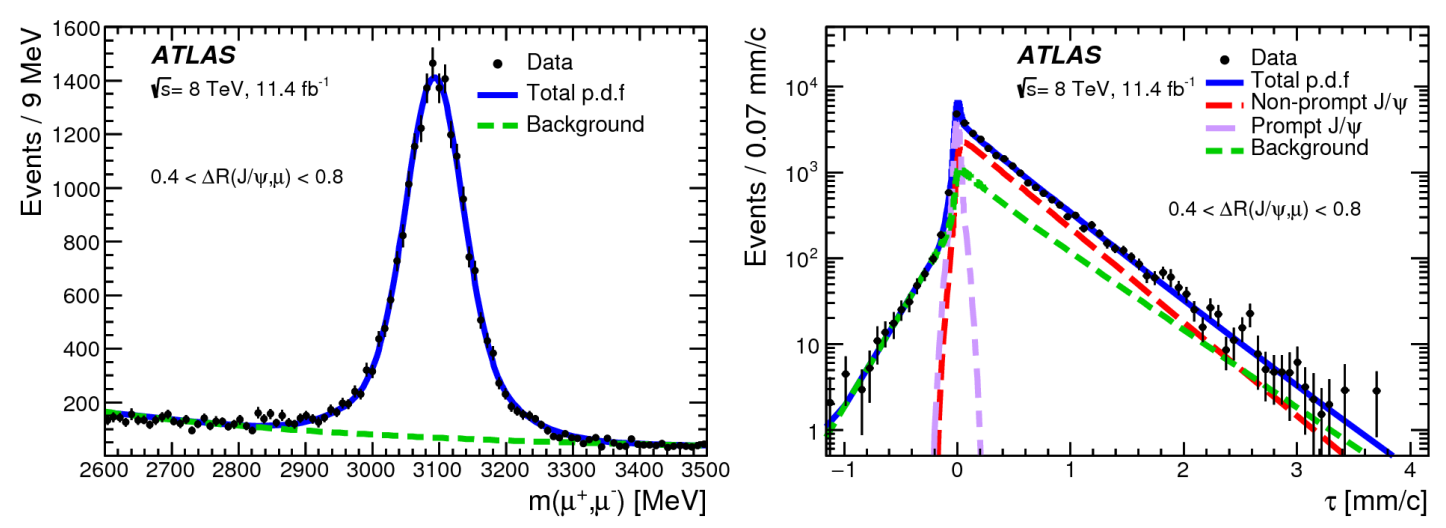

Figure 1: The di-muon mass and pseudo lifetime distribution of $J / \psi \rightarrow \mu \mu$ decay candidates. Superimposed are the fit projections [4].
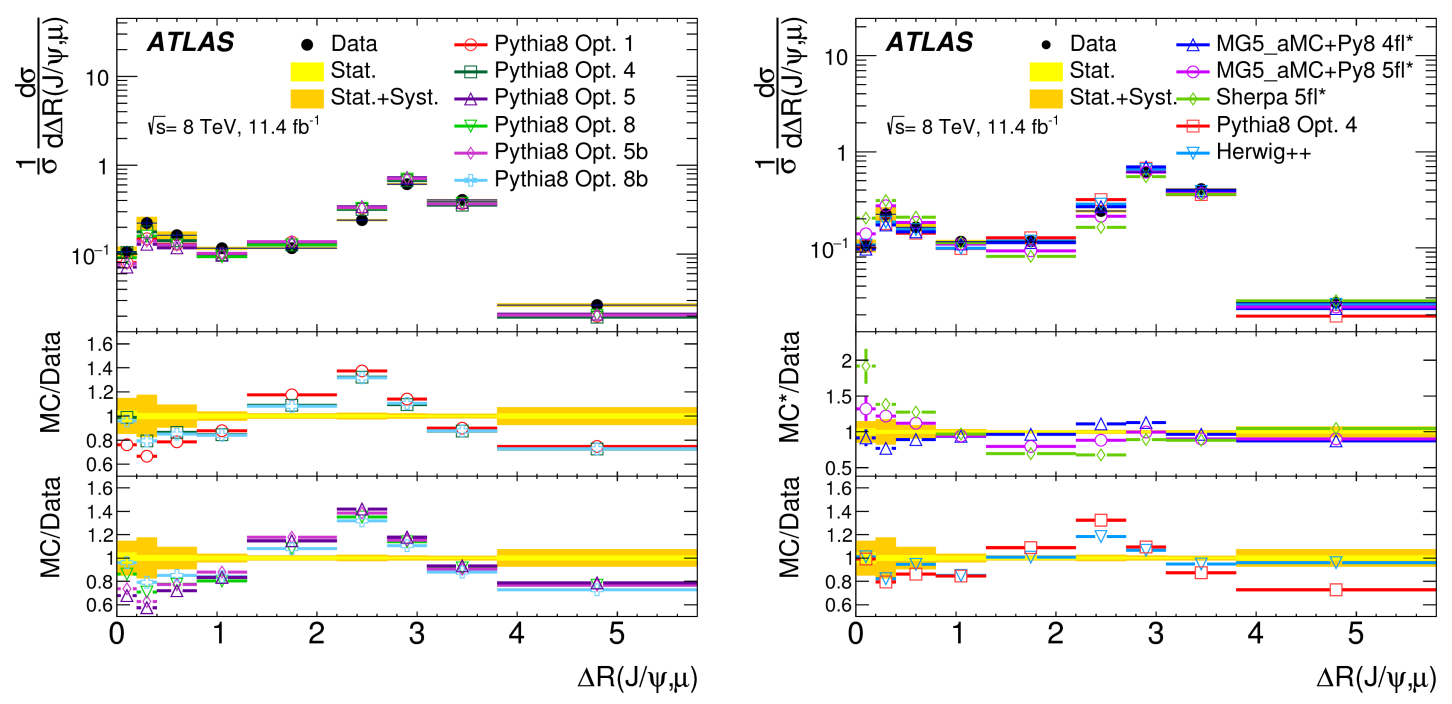

Figure 2: Differential $b \bar{b}$ cross section vs $\Delta R(J / \psi, \mu)$, normalised. The details of the splitting parameters are given in [4].

ATLAS has measured quarkonium production at centre of mass energies of $\sqrt{s}=7,8,13 \mathrm{TeV}[6$, 7] and more recently at $5.02 \mathrm{TeV}$ [8]. Shown in Fig. 3 are the differential prompt $J / \psi \rightarrow \mu^{+} \mu^{-}$and $\psi(2 S) \rightarrow \mu^{+} \mu^{-}$cross sections. The measurement is in good agreement with next-to-leading-order (NLO) non-relativistic QCD (NRQCD) predictions $[9,10]$.

\section{2 $\mathrm{X}(3872)$ production}

The exotic $X(3872)$ was first discovered by the Belle collaboration in 2003 and has since then been confirmed by many experiments. The quantum numbers have been firmly established, but the exact nature of this charm state is still unknown. ATLAS measured the production of $X(3872)$ in 

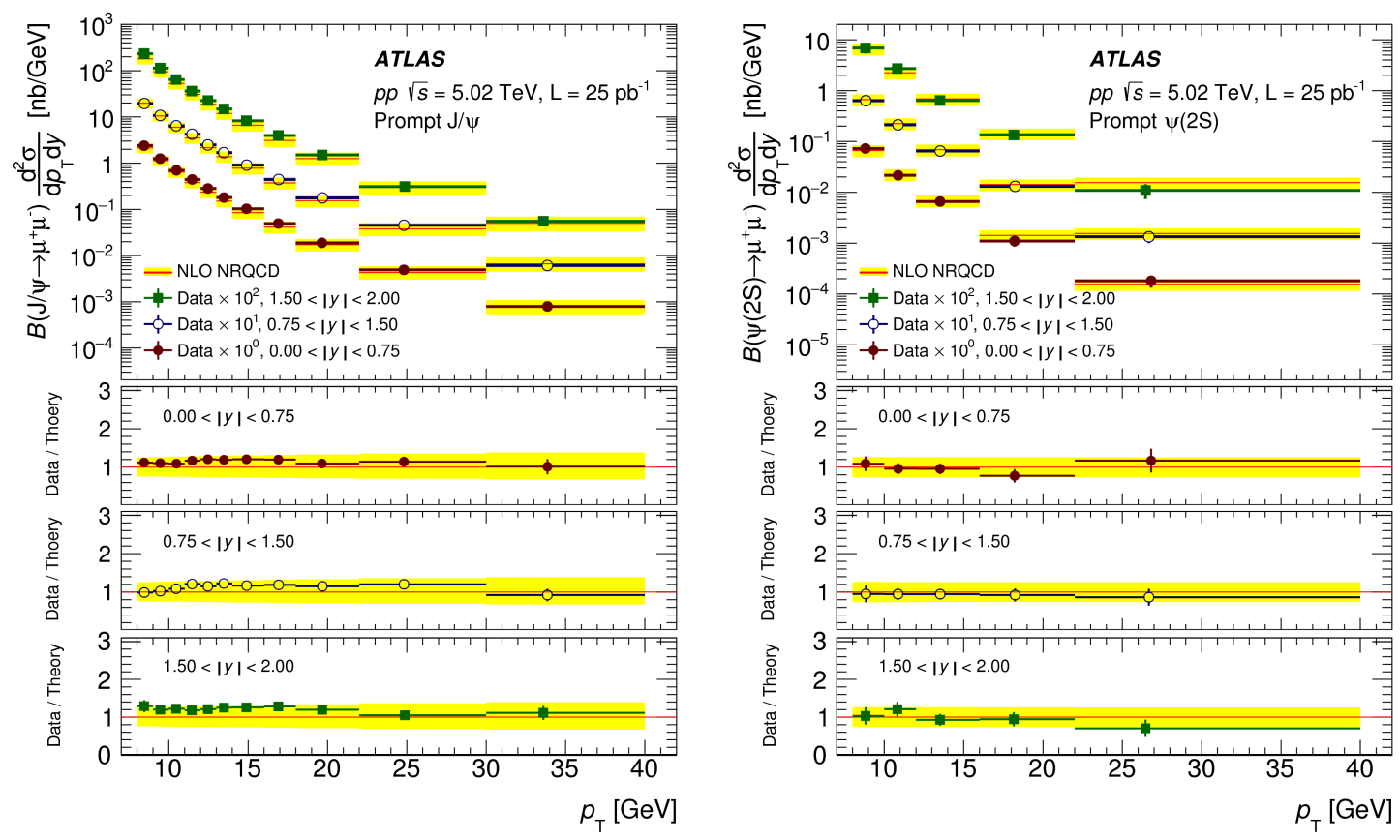

Figure 3: Differential prompt $J / \psi \rightarrow \mu^{+} \mu^{-}$and $\psi(2 S) \rightarrow \mu^{+} \mu^{-}$cross sections [8]. Overlaid are NLO NRQCD predictions based on long-distance matrix elements $[9,10]$.

the decay mode $X(3872) \rightarrow J / \psi \pi^{+} \pi^{-}$using $\psi(2 S) \rightarrow J / \psi \pi^{+} \pi^{-}$as normalisation [11]. Again the lifetime distribution is used to separate prompt and non-prompt production. A two lifetime fit including a long-lived and a short-lived component is employed. The short-lived fraction dominated by $B_{c}$ decays is measured to be:

$$
\frac{\sigma\left(p p \rightarrow B_{c}+X\right) \mathscr{B}\left(B_{c} \rightarrow X(3872)+X\right)}{\sigma(p p \rightarrow \text { non-prompt } X(3872)+X)}=(25 \pm 13(\text { stat }) \pm 2(\text { sys }) \pm 5(\text { spin })) \% .
$$

The fitted production ratios between $X(3872)$ and $\psi(2 S)$ are shown in Fig. 4. The prompt production cross section agrees well with the NLO NRQCD prediction [12]. The fixed-order-nextto-leading-log (FONLL) prediction of the non-prompt $X$ (3872) cross section [13] lies consistently above the data.

The di-pion invariant mass spectra in the $\rightarrow J / \psi \pi^{+} \pi^{-}$decay, shown in Fig. 5, contain information about intermediate decay particles. For the $\psi(2 S) \rightarrow J / \psi \pi^{+} \pi^{-}$decay this spectrum follows a Voloshin-Zakharov distribution $\frac{1}{\Gamma} \frac{d \Gamma}{d m_{\pi \pi}} \propto\left(m_{\pi \pi}^{2}-\lambda m_{\pi}^{2}\right)^{2} \times$ phase - space $)$. For the $X(3872) \rightarrow$ $J / \psi \pi^{+} \pi^{-}$the spectrum is consistent with the simulation of the decay through an intermediate $\rho^{0}$.

\subsection{Prompt $J / \psi$ pair production}

$J / \psi$ pairs can be produced in proton-proton collisions either by single parton scattering (SPS) or by double parton scattering (DPS) via two independent parton-parton interactions. The study of these interactions allows a better understanding of higher order and non-perturbative QCD.

ATLAS performed a measurement at $\sqrt{s}=8 \mathrm{TeV}$ using $11.4 \mathrm{fb}^{-1}$ of data [14]. Prompt $J / \psi$ events are separated from non-prompt source via the displaced secondary vertex and a lifetime fit. 

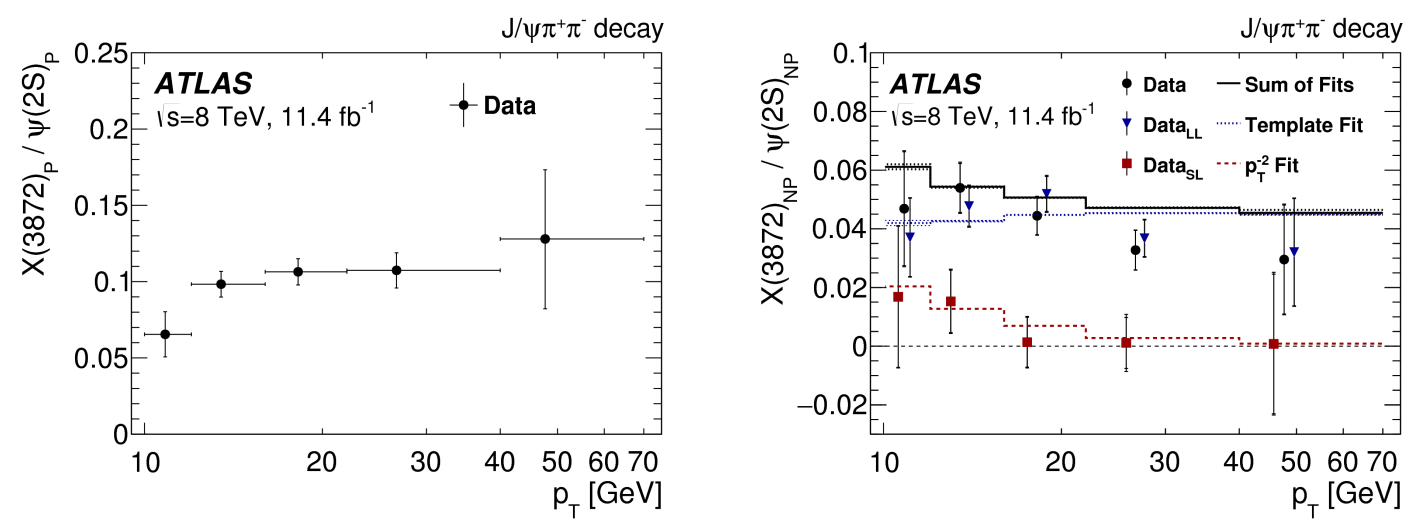

Figure 4: The ratio of $\sigma \times \mathscr{B} \frac{X(3872)}{\psi(2 S)}$ from a) prompt (P) and b) non-prompt (NP) production with a shortlifetime (SL) and a long-lifetime (LL) component [11]. The short-lived component is fitted with a $a / p_{T}^{2}$ function.
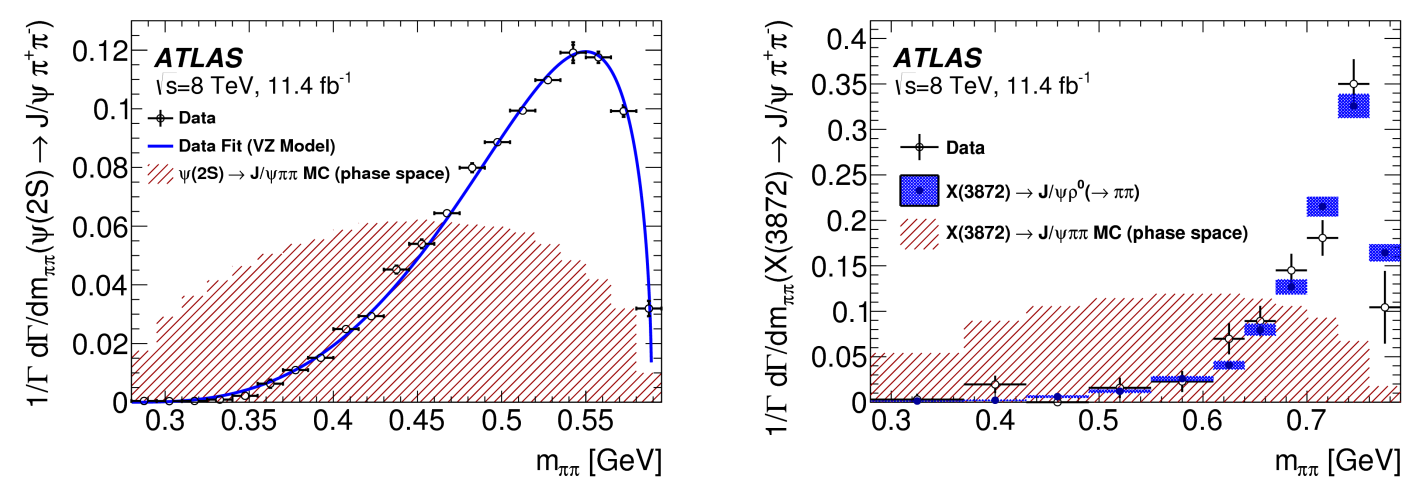

Figure 5: The differential decay width binned in the pion-pion invariant mass $m_{\pi \pi}$ for a) $\psi(2 S) \rightarrow$ $J / \psi \pi^{+} \pi^{-}$and b) $X(3872) \rightarrow J / \psi \pi^{+} \pi^{-}[11]$.

The phase space separates to qualitative regions: the away region at low $p_{T}$ of the di- $J / \psi$ system with the $J / \psi^{\prime} s$ produced back to back and high $p_{T}$ forward region, where the boosted $J / \psi^{\prime} s$ are close together. Using $J / \psi$ reconstructed from two independent events as template for DPS, the DPS fraction $f_{D P S}=(9.2 \pm 2.1$ (stat) $\pm 0.5($ syst $)) \%$ as well as the DPS cross section can be extracted and an effective cross section:

$$
\sigma_{e f f}=\frac{1}{2} \frac{\sigma_{J / \psi}^{2}}{\sigma_{D P S}^{J / \psi J / \psi}}=\frac{1}{2} \frac{\sigma_{J / \psi}^{2}}{f_{D P S} \times \sigma_{J / \psi J} / \psi}=6.3 \pm 1.6(\text { stat }) \pm 1.0(\text { syst }) \pm 0.1(B F) \pm 0.1(\text { lumi }) \mathrm{mb}
$$

can be measured. Comparing with other experiments, effective cross sections extracted from diquarkonium states seem to be lower than those measured for other final states.

The total differential di- $J / \psi$ cross section and the estimated DPS contribution are shown in Fig. 6. The data seem in reasonable agreement with leading-order DPS [15] and next-to-leadingorder colour singlet non-relativistic QCD calculations without loops [16, 17]. 

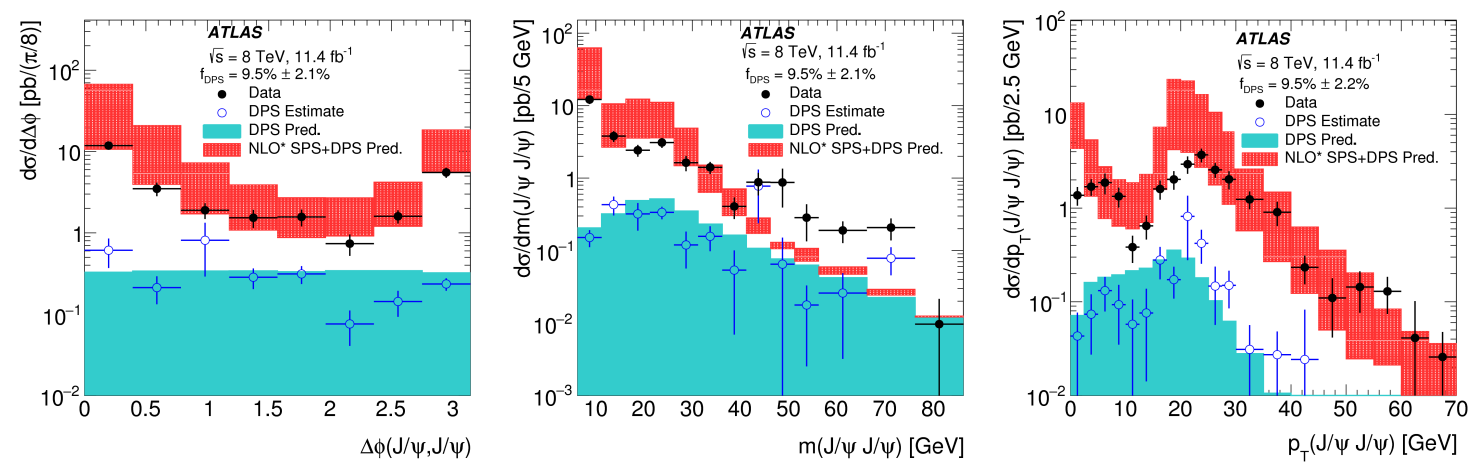

Figure 6: Total and double parton scattering (DPS) differential cross sections versus azimuthal opening angle between the two $J / \psi^{\prime} s$, invariant $J / \psi-J / \psi$ mass and transverse momentum of the di- $J / \psi$ system [14]. Overlaid are the predictions from leading-order DPS [15] and next-to-leading-order colour singlet non-relativistic QCD calculations without loops [16, 17].

\section{Conclusion}

The ATLAS experiment at the LHC has continuously performed measurements of $b \bar{b}$ and charmonium production of $J / \psi, \psi(2 S)$ and exotic $X(3872)$ states. These studies now include also rarer processes like di- $J / \psi$ production. Such investigations of Heavy Flavour production provide important tests of Quantum Chromodynamics. The measurements are overall in good agreement with theoretical predictions and in several cases have been used to tune Monte Carlo Generators.

\section{References}

[1] ATLAS Collaboration, "The ATLAS Experiment at the CERN Large Hadron Collider," JINST 3 (2008) S08003.

[2] L. Evans and P. Bryant, "LHC Machine," JINST 3 (2008) S08001.

[3] ATLAS Collaboration, "Measurement of the $b \bar{b}$ dijet cross section in pp collisions at $\sqrt{s}=7 \mathrm{TeV}$ with the ATLAS detector," Eur. Phys. J. C 76 (2016) no.12, 670 [arXiv:1607.08430 [hep-ex]].

[4] ATLAS Collaboration, "Measurement of $b$-hadron pair production with the ATLAS detector in proton-proton collisions at $\sqrt{s}=8 \mathrm{TeV}$," JHEP 1711 (2017) 062 [arXiv:1705.03374 [hep-ex]].

[5] T. Sjostrand, S. Mrenna and P. Z. Skands, "A Brief Introduction to PYTHIA 8.1," Comput. Phys. Commun. 178 (2008) 852 [arXiv:0710.3820 [hep-ph]].

[6] ATLAS Collaboration, "Measurement of the differential cross-sections of prompt and non-prompt production of $J / \psi$ and $\psi(2 S)$ in $p p$ collisions at $\sqrt{s}=7$ and $8 \mathrm{TeV}$ with the ATLAS detector," Eur. Phys. J. C 76 (2016) no.5, 283 [arXiv:1512.03657 [hep-ex]].

[7] ATLAS Collaboration, "Measurement of the differential non-prompt $J / \psi$ production fraction in $\sqrt{s}=$ $13 \mathrm{TeV}$ pp collisions at the ATLAS experiment," ATLAS-CONF-2015-030.

[8] ATLAS Collaboration, "Measurement of quarkonium production in proton-lead and proton-proton collisions at 5.02 TeV with the ATLAS detector," Eur. Phys. J. C 78 (2018) no.3, 171 [arXiv:1709.03089 [nucl-ex]]. 
[9] Y. Q. Ma, K. Wang and K. T. Chao, “ $J / \psi\left(\psi^{\prime}\right)$ production at the Tevatron and LHC at $\mathscr{O}\left(\alpha_{s}^{4} v^{4}\right)$ in nonrelativistic QCD,” Phys. Rev. Lett. 106 (2011) 042002 [arXiv:1009.3655 [hep-ph]].

[10] H. S. Shao, H. Han, Y. Q. Ma, C. Meng, Y. J. Zhang and K. T. Chao, "Yields and polarizations of prompt $J / \psi$ and $\psi(2 S)$ production in hadronic collisions," JHEP 1505 (2015) 103 [arXiv:1411.3300 [hep-ph]].

[11] ATLAS Collaboration, "Measurements of $\psi(2 S)$ and $X(3872) \rightarrow J / \psi \pi^{+} \pi^{-}$production in $p p$ collisions at $\sqrt{s}=8 \mathrm{TeV}$ with the ATLAS detector," JHEP 1701 (2017) 117 [arXiv:1610.09303 [hep-ex]].

[12] C. Meng, H. Han and K. T. Chao, “X(3872) and its production at hadron colliders," Phys. Rev. D 96 (2017) no.7, 074014 [arXiv:1304.6710 [hep-ph]].

[13] M. Cacciari, S. Frixione, N. Houdeau, M. L. Mangano, P. Nason and G. Ridolfi, "Theoretical predictions for charm and bottom production at the LHC," JHEP 1210 (2012) 137 [arXiv:1205.6344 [hep-ph]].

[14] ATLAS Collaboration, "Measurement of the prompt $\mathrm{J} / \psi$ pair production cross-section in $\mathrm{pp}$ collisions at $\sqrt{s}=8 \mathrm{TeV}$ with the ATLAS detector," Eur. Phys. J. C 77 (2017) no.2, 76 [arXiv:1612.02950 [hep-ex]].

[15] C. Borschensky and A. Kulesza, "Double parton scattering in pair production of $J / \psi$ mesons at the LHC revisited,” Phys. Rev. D 95 (2017) no.3, 034029 [arXiv:1610.00666 [hep-ph]].

[16] J. P. Lansberg and H. S. Shao, "Production of $J / \psi+\eta_{c}$ versus $J / \psi+J / \psi$ at the LHC: Importance of Real $\alpha_{s}^{5}$ Corrections,” Phys. Rev. Lett. 111 (2013) 122001 [arXiv:1308.0474 [hep-ph]].

[17] J. P. Lansberg and H. S. Shao, “ $J / \psi$-pair production at large momenta: Indications for double parton scatterings and large $\alpha_{s}^{5}$ contributions,” Phys. Lett. B 751 (2015) 479 [arXiv:1410.8822 [hep-ph]]. 